Martin:

Small Ball Asymptotics for the Stochastic Wave Equation

Sonderforschungsbereich 386, Paper 360 (2003)

Online unter: http://epub.ub.uni-muenchen.de/

Projektpartner
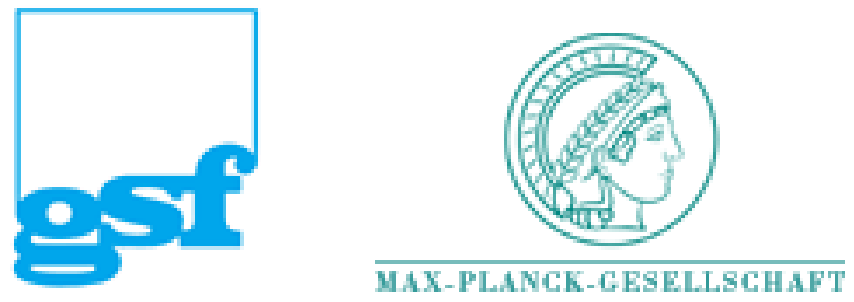


\title{
Small Ball Asymptotics for the Stochastic Wave Equation *
}

\author{
Andreas Martin ${ }^{\dagger}$
}

January 21, 2003

\begin{abstract}
We examine the small ball asymptotics for the weak solution $X$ of the stochastic wave equation

$$
\frac{\partial^{2} X}{\partial t^{2}}(t, x)-\frac{\partial^{2} X}{\partial x^{2}}(t, x)=g(X(t, x))+f(t, x) d W(t, x)
$$

on the real line with deterministic initial conditions.
\end{abstract}

Key words: small ball, stochastic wave equation, Brownian sheet.

\section{Introduction}

Let $X$ be a random variable on a probability space $(\Omega, \mathcal{F}, \mathbb{P})$ taking values in a real Banach space. The small ball asymptotics is the order of the probability $\mathbb{P}\{\|X\|<\varepsilon\}$ as $\varepsilon \rightarrow 0+$. We examine the small ball asymptotics for the weak solution $X=\left\{X(t, x) ;(t, x) \in \mathbb{R}_{+} \times \mathbb{R}\right\}$ of the stochastic wave equation

$$
\frac{\partial^{2} X}{\partial t^{2}}(t, x)-\frac{\partial^{2} X}{\partial x^{2}}(t, x)=g(X(t, x))+f(t, x) d W(t, x)
$$

on the real line with deterministic initial conditions $(F, \mu)$. The random field $X$ is almost surely continuous and for each compact set $A \subset \mathbb{R}_{+} \times \mathbb{R}, X_{\mid A}$ can be considered as a random variable with values in the Banach space of continuous functions on $A$ endowed with the supremum norm. In the case $g=0$ and $f=1$ the solution of (1) is very similar to a Brownian

${ }^{*}$ AMS Subject Classification. Primary 60H15; Secondary 60G60.

This work was partly supported by a DAAD Doktorandenstipendium im Rahmen des gemeinsamen Hochschulsonderprogramms III von Bund und Ländern.

${ }^{\dagger}$ Institute of Biomathematics and Biometry, GSF-National Research Centre for Environment and Health, Ingolstädter Landstraße 1, 85764 Neuherberg/München, Germany. E-mail: martin@gsf.de 
sheet. M. TALAGRAND derived in [12] precise small ball asymptotics for this random field. This is our point of depart, and as a matter of fact, the small ball asymptotics for the solution of (1) is of the same order: Let $f$ be a square integrable function which is continuous and does not vanish at at least one point. Furthermore, let $g$ be LiPsChitz continuous and $u$ be the solution of the deterministic partial differential equation

$$
\frac{\partial^{2} u}{\partial t^{2}}(t, x)-\frac{\partial^{2} u}{\partial x^{2}}(t, x)=g(u(t, x))
$$

with initial conditions $(F, \mu)$. Theorem 2.11 below states in terms of Landau symbols that the upper small ball asymptotics reads

$$
\mathbb{P}\left\{\sup _{\triangle(r, 0)}|X(t, x)-u(t, x)|<\varepsilon\right\} \leq \mathcal{O}\left(\exp \left(-(\log (1 / \varepsilon))^{3} / \varepsilon^{2}\right)\right)
$$

with $\triangle(r, 0)=\{(s, y): 0 \leq s \leq r,|y| \leq r-s\}$. Moreover, Theorem 3.4 states that the lower asymptotics is of the same order.

The rate of the asymptotics is given in terms of Landau symbols. Let $u$ and $v$ be real valued functions. We write $u(x)=\mathcal{O}(v(x))$, if there is a constant $C$ such that $|u(x)| /|v(x)|<C$ for $x \rightarrow x_{0}$ or $x \rightarrow \infty$. Furthermore, let $w$ be a real valued function. If there are functions $u$ and $v$ with $w(x) \leq$ $u(x)$ for $x \rightarrow x_{0}$ or $x \rightarrow \infty$ and $u(x)=\mathcal{O}(v(x))$, then we write $w(x) \leq$ $\mathcal{O}(v(x))$. Vice versa, if there are functions $u$ and $v$ with $w(x) \geq u(x)$ for $x \rightarrow x_{0}$ or $x \rightarrow \infty$ and $u(x)=\mathcal{O}(v(x))$, then we write $w(x) \geq \mathcal{O}(v(x))$.

J. KuELBS, W.V. Li and W. LINDE showed that evaluating small ball asymptotics are in general equivalent to solving a demanding metric entropy problem in functional analysis; see [6] and [9]. This explains why relatively little is known about small ball asymptotics, except for special examples. The oldest examples concern Brownian motions and Brownian bridges. Let $B M$ denote a standard Brownian motion and $B B$ a Brownian bridge. Then

$$
\mathbb{P}\left\{\sup _{t \in[0,1]}|B M(t)|<\varepsilon\right\} \sim \frac{4}{\pi} \exp \left\{-\frac{\pi^{2}}{8 \varepsilon^{2}}\right\}
$$

and the probability

$$
\mathbb{P}\left\{\sup _{t \in[0,1]}|B B(t)|<\varepsilon\right\} \sim \frac{\sqrt{2 \pi}}{\varepsilon} \exp \left\{-\frac{\pi^{2}}{8 \varepsilon^{2}}\right\}
$$

for $\varepsilon \rightarrow 0+$, respectively. For the proof of these and many more interesting results and topics related to small ball asymptotics we refer to the surveys J.P.R. Christensen [3], M. Ledoux [7], M.A. Lifshits [11] and W.V. Li and Q-M. SHAO [10].

J.B. WALSH introduced in the eighties an integration theory with respect to martingale measures in order to establish a framework for stochastic partial differential equations similar to ITÔ's theory, see for example [13]. It was 
used by R.A. CARMonA and D. NuAlart in 1988 to examine the vibrating string in [1] and its companion article [2]. Different approaches to stochastic partial differential equations are treated in the textbooks [4] of G. DAPRATO and J. ZABCZYK in 1992 and [5] of H. Holden, B. ØKsendal, J. UBøE and T. ZHANG in 1996.

This article is organised as follows. The upper asymptotics are derived in Section 2 and the lower ones are obtained in Section 3. The underlying ideas are similar. We transfer the small ball asymptotics for the Brownian sheet to the solution $X$ of (1) in the case $g=0$ and $f=1$ by a clockwise rotation of $45^{\circ}$. Then we use the partial differential equation to derive the asymptotics for the general case. In each section we discuss as a special example the KLEIN-GORDON equation on the real line

$$
\frac{\partial^{2} X}{\partial t^{2}}(t, x)-\frac{\partial^{2} X}{\partial x^{2}}(t, x)-\alpha X(t, x)=d W(t, x)
$$

perturbed by space-time white noise.

\section{Upper Asymptotics}

We start with preparations for the proofs of Proposition 2.6 and its Corollary 2.7. The underlying ideas are similar to the ones used in the proof of the upper asymptotics in Theorem 1.1 [12]. A central tool is TALAGRAND's combinatorial inequality stated in Proposition 2.5.

Proposition 2.1 Let $C \in(0,1]$ and let $M, N \in \mathbb{N}$ with $C M \leq N \leq$ $M$. Then for a sequence $\left(\xi_{i}\right)_{i \leq N}$ of independent standard normal random variables one has

$$
\mathbb{P}\left\{\sum_{i \leq N}\left|\xi_{i}\right| \leq \frac{M}{C_{0}}\right\} \leq \exp \left(-\frac{M}{C_{0}}\right),
$$

where $C_{0}=-2 /\left(C \ln \mathbb{E}\left\{\exp \left(-\left|\xi_{1}\right|\right)\right\}\right)$.

Proof. Chebyshev's exponential inequality yields

$$
\mathbb{P}\left\{\sum_{i \leq N}\left|\xi_{i}\right| \leq \frac{M}{C_{0}}\right\} \leq \exp \left(\frac{M}{C_{0}}\right) \mathbb{E}\left\{\exp \left(-\sum_{i \leq N}\left|\xi_{i}\right|\right)\right\} .
$$

Since the random variables $\xi_{i}$ are independent and identically distributed the right hand side of $(2)$ is equal to

$$
\exp \left(-\frac{C M \ln \mathbb{E}\left\{\exp \left(-\left|\xi_{1}\right|\right)\right\}}{2}\right) \mathbb{E}\left\{\exp \left(-\left|\xi_{1}\right|\right)\right\}^{N}=\mathbb{E}\left\{\exp \left(-\left|\xi_{1}\right|\right)\right\}^{N-C M / 2}
$$


Moreover, $\mathbb{E}\left\{\exp \left(-\left|\xi_{1}\right|\right)\right\}<1$ and $N \geq C M$, thus one has

$$
\mathbb{E}\left\{\exp \left(-\left|\xi_{1}\right|\right)\right\}^{N-C M / 2} \leq \mathbb{E}\left\{\exp \left(-\left|\xi_{1}\right|\right)\right\}^{C M / 2}=\exp \left(-\frac{M}{C_{0}}\right)
$$

which completes the proof.

Let $H=L^{2}\left([0,1]^{2}, \mathcal{B}[0,1]^{2}, \lambda^{2}\right)$ and let $\left(b_{m}\right)$ be a complete orthonormal system of (the separable Hilbert space) $H$. Let further $\left(\xi_{m}\right)$ be a sequence of independent standard normal random variables. An isonormal process $W=\{W(h) ; h \in H\}$ can be constructed by

$$
W(h)=\sum_{m} \xi_{m}\left\langle h, b_{m}\right\rangle,
$$

where the brackets are the canonical inner product of $H$; see for example [4]. This construction is independent of the choice of the complete orthonormal system, because the process is determined by its finite dimensional distributions. On the other hand, the proof of TALAGRAND's combinatorial inequality (5) depends heavily on the choice of a special complete orthonormal system which will be introduced now.

Definition 2.2 Set $h_{-1,0} \equiv 1$,

$$
h(u)=\left\{\begin{aligned}
1 & \text { if } u \in[0,1 / 4) \cup(3 / 4,1] \\
-1 & \text { if } u \in[1 / 4,3 / 4)
\end{aligned}\right.
$$

and fix an integer $q \geq 1$. Define for integers $m \geq 0$ and $0 \leq k<2^{q m}$

$$
h_{m, k}(u)=2^{q m / 2} h\left(2^{q m}\left(u-k 2^{-q m}\right)\right) .
$$

Furthermore, set

$$
\mathcal{I}=\left\{(m, k) ; m \geq 0,0 \leq k<2^{q m}\right\} \cup\{(-1,0)\}
$$

and

$$
\mathcal{O S}(\mathcal{I})=\left\{h_{m, k} \otimes h_{n, l} ;((m, k),(n, l)) \in \mathcal{I} \times \mathcal{I}\right\} .
$$

The family $\mathcal{O S}(\mathcal{I})$ is for each $q \geq 1$ a complete orthonormal system in $H$.

We need the following two sets of indices for the further discussion. Notice that $A^{o}=\bar{A} \backslash \partial A$ denotes the open interior of a set $A$ and $\operatorname{supp}(f)$ denotes the support of a function $f$.

Definition 2.3 For a set $A \subset[0,1]^{2}$ define

$$
\mathcal{I}(A)=\left\{((m, k),(n, l)) \in \mathcal{I} \times \mathcal{I} ; \operatorname{supp}\left(h_{m, k} \otimes h_{n, l}\right)^{o} \subset A\right\} .
$$

For an integer $N \in \mathbb{N}$ define

$$
\begin{aligned}
& \mathcal{T}(N)=\{((m, k),(n, l)) \in \mathcal{I} \times \mathcal{I} \quad ; \quad m \geq 0, n \geq 1, m+n=N, \\
&\left.0 \leq k<2^{q m}, 0 \leq l<2^{q n}\right\} .
\end{aligned}
$$


The set $\mathcal{I}(A)$ is that set of indices in $\mathcal{I} \times \mathcal{I}$ for which the corresponding functions in $\mathcal{O S}(\mathcal{I})$ have their supports essentially in $A$. The set $\mathcal{T}(N)$ consists of those indices such that the corresponding functions in $\mathcal{O S}(\mathcal{I})$ have supports with given and fixed length of the edges. In the next example the relation between $\mathcal{I}(A)$ and $\mathcal{T}(N)$ is exploited.

Example 2.4 Let $A=\operatorname{supp}\left(h_{m, k} \otimes h_{n, l}\right)$ for fixed integers $m \geq 0, n \geq 1$, $0 \leq k<2^{q m}$ and $0 \leq l<2^{q n}$. If $N<m+n$ then the sets $\mathcal{I}(A)$ and $\mathcal{T}(N)$ are disjoint, because the edges of the supports of functions associated to $\mathcal{T}(N)$ are too long. If $N \geq m+n$, however, then $\mathcal{I}(A) \cap \mathcal{T}(N)$ is not empty and the number of elements $|\mathcal{I}(A) \cap \mathcal{T}(N)|=(N-(m+n)+1) 2^{q(N-(m+n))}$. It is related to $|\mathcal{T}(N)|$ by

$$
\begin{aligned}
& \frac{|\mathcal{I}(A) \cap \mathcal{T}(N)|}{|\mathcal{T}(N)|}=\frac{(N-(m+n)+1) 2^{q(N-(m+n))}}{N 2^{q N}} \\
& \quad=\left(1-\frac{m+n}{N}+\frac{1}{N}\right) 2^{-q(m+n)} \geq \frac{2^{-q(m+n)}}{m+n}=: C>0 .
\end{aligned}
$$

Thus for all $N \geq m+n$ there is a constant $C>0$ independent of $N$ such that

$$
|\mathcal{T}(N)| \geq|\mathcal{I}(A) \cap \mathcal{T}(N)| \geq C|\mathcal{T}(N)|
$$

The next proposition is the key to the proof of Proposition 2.6. Define for $(m, k) \in \mathcal{I}$

$$
g_{m, k}(x)=\int_{0}^{x} h_{m, k}(u) d u .
$$

Proposition 2.5 ([12], Proposition 2.4) For $q=9, N \in \mathbb{N}$, and for each family of numbers $\left(a_{((m, k),(n, l))}\right)_{\mathcal{T}(N)}$ one has

$$
\sup _{x, y \in[0,1]} \sum_{\mathcal{T}(N)} a_{((m, k),(n, l))} g_{m, k}(x) g_{n, l}(y) \geq 2^{-3 q N / 2-7} \sum_{\mathcal{T}(N)}\left|a_{((m, k),(n, l))}\right| .
$$

One idea of the proof of this proposition is that the $g_{m, k} \otimes g_{n, l}$ s have positive and negative values and therefore can compensate the sign of the numbers $a_{((m, k),(n, l))}$. Another ingredient is the fact that for fixed $m$ and $n$ and different pairs $(k, l)$ and $\left(k^{\prime}, l^{\prime}\right)$ the supports of $g_{m, k} \otimes g_{n, l}$ and $g_{m, k^{\prime}} \otimes g_{n, l^{\prime}}$ have disjoint interiors.

Define for $x, y \in[0,1]$ the set $R(x, y)=[0, x] \times[0, y]$.

Proposition 2.6 Suppose that $f \in L^{2}[0,1]^{2}$ satisfies the decomposition

$$
f=f_{c} \cdot 1_{\operatorname{supp}\left(h_{m^{\prime}, k^{\prime}} \otimes h_{n^{\prime}, l^{\prime}}\right)}+f_{v} \cdot 1_{\operatorname{supp}\left(h_{m^{\prime}, k^{\prime}} \otimes h_{n^{\prime}, l^{\prime}}\right)^{c}},
$$


where $f_{c} \in \mathbb{R} \backslash\{0\}, f_{v} \in L^{2}[0,1]^{2}$ and $h_{m^{\prime}, k^{\prime}} \otimes h_{n^{\prime}, l^{\prime}} \in \mathcal{O} \mathcal{S}(\mathcal{I})$. Moreover, let $\left\{W(h) ; h \in L^{2}[0,1]^{2}\right\}$ be an isonormal process. Then for $\varepsilon \rightarrow 0+$

$$
\mathbb{P}\left\{\sup _{x, y \in[0,1]}\left|W\left(1_{R(x, y)} f\right)\right|<\varepsilon\right\} \leq \mathcal{O}\left(\exp \left(-\frac{\left|f_{c}\right|^{2}(\log (1 / \varepsilon))^{3}}{C^{3} \varepsilon^{2}}\right)\right),
$$

where $C$ is a positive constant depending only on $m^{\prime}+n^{\prime}$.

Notice that the asymptotics depend only on a constant part of $f$.

Before we begin to prove Proposition 2.6 we need another fact. Let $f \in$ $L^{2}[0,1]^{2}$ and let $\left(b_{m}\right)_{m \in \mathbb{N}}$ and $\left(\xi_{m}\right)_{m \in \mathbb{N}}$ be like in the remarks to equation (3). Furthermore, let $\mathcal{K}, \mathcal{L} \subset \mathbb{N}$ with $\mathcal{K} \cap \mathcal{L}=\emptyset$. Then two stochastic processes $W_{\mathcal{K}}$ and $W_{\mathcal{L}}$ are given by

$$
W_{\mathcal{K}}=\left\{W_{\mathcal{K}}\left(1_{R(x, y)} f\right)=\sum_{m \in \mathcal{K}} \xi_{m}\left\langle 1_{R(x, y)} f, b_{m}\right\rangle ;(x, y) \in[0,1]^{2}\right\}
$$

and

$$
W_{\mathcal{L}}=\left\{W_{\mathcal{L}}\left(1_{R(x, y)} f\right)=\sum_{m \in \mathcal{L}} \xi_{m}\left\langle 1_{R(x, y)} f, b_{m}\right\rangle ;(x, y) \in[0,1]^{2}\right\} .
$$

Recall that a regular random variable $X$ with values in a Banach space $B$ is Gaussian, if for each continuous linear functional $l$ on $B$ the random variable $l(X)$ is a real Gaussian random variable; see [8], p. 55. Therefore $W_{\mathcal{K}}$ and $W_{\mathcal{L}}$ can be considered as centred Gaussian random variables with values in $\left(C[0,1]^{2},\|\cdot\|_{\infty}\right)$. Moreover they are independent and Lemma 2.1, [12] implies that

$$
\mathbb{P}\left\{\left\|W_{\mathcal{K}}+W_{\mathcal{L}}\right\|_{\infty}<\varepsilon\right\} \leq \mathbb{P}\left\{\left\|W_{\mathcal{K}}\right\|_{\infty}<\varepsilon\right\}
$$

for any $\varepsilon>0$.

Proof of Proposition 2.6. We construct the stochastic process $W=$ $\left\{W(h) ; h \in L^{2}[0,1]^{2}\right\}$ explicitly. Consider the complete orthonormal system $\mathcal{O S}(\mathcal{I})$, a sequence $\left(\xi_{m, k, n, l}\right)$ of independent standard normal random variables and let $W$ be given by

$$
W(h)=\sum_{\mathcal{I} \times \mathcal{I}} \xi_{m, k, n, l}\left\langle h, h_{m, k} \otimes h_{n, l}\right\rangle .
$$

Set $A=\operatorname{supp}\left(h_{m^{\prime}, k^{\prime}} \otimes h_{n^{\prime}, l^{\prime}}\right)$. Then by construction of $W$ and the assumption on $f$ one has

$$
\begin{aligned}
& \mathbb{P}\left\{\sup _{x, y \in[0,1]}\left|W\left(1_{R(x, y)} f\right)\right|<\varepsilon\right\} \\
& \quad=\mathbb{P}\left\{\sup _{x, y \in[0,1]}\left|\sum_{\mathcal{I} \times \mathcal{I}} \xi_{m, k, n, l}\left\langle 1_{R(x, y)}\left(f_{c} 1_{A}+f_{v} 1_{A^{c}}\right), h_{m, k} \otimes h_{n, l}\right\rangle\right|<\varepsilon\right\}
\end{aligned}
$$




$$
\begin{aligned}
=\mathbb{P}\left\{\sup _{x, y \in[0,1]} \mid \sum_{\mathcal{I} \times \mathcal{I}} \xi_{m, k, n, l}\right. & \left(f_{c}\left\langle 1_{R(x, y)} 1_{A}, h_{m, k} \otimes h_{n, l}\right\rangle\right. \\
& \left.\left.+\left\langle f_{v} 1_{R(x, y)} 1_{A^{c}}, h_{m, k} \otimes h_{n, l}\right\rangle\right) \mid<\varepsilon\right\} .
\end{aligned}
$$

Only linearity of the inner product was used. Set $\mathcal{K}=\mathcal{I}(A)$ and $\mathcal{L}=$ $\mathcal{I} \times \mathcal{I} \backslash \mathcal{I}(A)$. Then by inequality (6) the probability in (7) is less or equal to

$$
\begin{array}{r}
\mathbb{P}\left\{\sup _{x, y \in[0,1]} \mid \sum_{\mathcal{I}(A)} \xi_{m, k, n, l}\left(f_{c}\left\langle 1_{R(x, y)} 1_{A}, h_{m, k} \otimes h_{n, l}\right\rangle\right.\right. \\
\left.\left.+\left\langle f_{v} 1_{R(x, y)} 1_{A^{c}}, h_{m, k} \otimes h_{n, l}\right\rangle\right) \mid<\varepsilon\right\} .
\end{array}
$$

The indices in $\mathcal{I}(A)$ refer only to functions in $\mathcal{O S}(\mathcal{I})$ with support essentially in $A$. Hence for $g_{m, k}$ defined like in (4) by $g_{m, k}(x)=\int_{0}^{x} h_{m, k}(u) d u$, the probability in (8) is equal to

$$
\mathbb{P}\left\{\sup _{x, y \in[0,1]}\left|\sum_{\mathcal{I}(A)} \xi_{m, k, n, l} f_{c} g_{m, k}(x) g_{n, l}(y)\right|<\varepsilon\right\} .
$$

The indices are further reduced from $\mathcal{I}(A)$ to $\mathcal{I}(A) \cap \mathcal{T}(N)$. Inequality (6) yields that the expression above is less or equal to

$$
\mathbb{P}\left\{\sup _{x, y \in[0,1]}\left|\sum_{\mathcal{I}(A) \cap \mathcal{T}(N)} \xi_{m, k, n, l} f_{c} g_{m, k}(x) g_{n, l}(y)\right|<\varepsilon\right\}
$$

and by TALAGRAND's inequality (5) this is bounded from above by

$$
\begin{aligned}
& \mathbb{P}\left\{\sum_{\mathcal{I}(A) \cap \mathcal{T}(N)}\left|\xi_{m, k, n, l} f_{c}\right|<2^{3 q N / 2+7} \varepsilon\right\} \\
& \quad=\mathbb{P}\left\{\sum_{I(A) \cap \mathcal{T}(N)}\left|\xi_{m, k, n, l}\right|<\left|f_{c}\right|^{-1} 2^{3 q N / 2+7} \varepsilon\right\} .
\end{aligned}
$$

The final part follows from Proposition 2.1. To this end recall that by Example 2.4 there is a constant $C>0$ which is independent of $N$ such that

$$
|\mathcal{T}(N)| \geq|\mathcal{I}(A) \cap \mathcal{T}(N)| \geq C|\mathcal{T}(N)| .
$$

Moreover, let $\xi$ be standard normal and set $C_{0}=-2 /(C \ln \mathbb{E}\{\exp (-|\xi|)\})$. Let $\varepsilon$ be sufficiently small such that for at least one $N \geq m^{\prime}+n^{\prime}$,

$$
\left|f_{c}\right|^{-1} \varepsilon \leq \frac{N 2^{q N}}{C_{0} 2^{3 q N / 2+7}}=\frac{N 2^{-q N / 2} 2^{-7}}{C_{0}} .
$$

Choose $N$ maximal; $N$ is of order $\log (1 / \varepsilon)$. Then (9) is less or equal to

$$
\mathbb{P}\left\{\sum_{\mathcal{I}(A) \cap \mathcal{T}(N)}\left|\xi_{m, k, n, l}\right|<\frac{N 2^{q N}}{C_{0}}\right\},
$$


and by Proposition 2.1 this probability can be bounded from above by

$$
\exp \left(-\frac{N 2^{q N}}{C_{0}}\right)=\exp \left(-\frac{N^{3} 2^{-14}}{C_{0}^{3}\left(\frac{N 2^{-q N / 2} 2^{-7}}{C_{0}}\right)^{2}}\right) .
$$

This bound is of order

$$
\exp \left(-\frac{\left|f_{c}\right|^{2}(\log (1 / \varepsilon))^{3}}{C_{0}^{3} \varepsilon^{2}}\right)
$$

In summary, for $\varepsilon \rightarrow 0+$,

$$
\mathbb{P}\left\{\sup _{x, y \in[0,1]}\left|W\left(1_{R(x, y)} f\right)\right|<\varepsilon\right\} \leq \mathcal{O}\left(\exp \left(-\frac{\left|f_{c}\right|^{2}(\log (1 / \varepsilon))^{3}}{C_{0}^{3} \varepsilon^{2}}\right)\right) .
$$

This completes the proof.

Proposition 2.6 can be generalized.

Corollary 2.7 Let $f \in L^{2}[0,1]^{2}$ and suppose that there are indices $\left(m^{\prime}, k^{\prime}\right)$, $\left(n^{\prime}, l^{\prime}\right) \in \mathcal{I}$ and a real number $f_{c}>0$ such that

$$
\left.\left(f^{2}-f_{c}\right)\right|_{\operatorname{supp}\left(h_{m^{\prime}, k^{\prime}} \otimes h_{n^{\prime}, l^{\prime}}\right)}>0
$$

almost everywhere, where $h_{m^{\prime}, k^{\prime}} \otimes h_{n^{\prime}, l^{\prime}} \in \mathcal{O} \mathcal{S}(\mathcal{I})$. Let $\left\{W(h) ; h \in L^{2}[0,1]^{2}\right\}$ be an isonormal process, then for $\varepsilon \rightarrow 0+$

$$
\mathbb{P}\left\{\sup _{x, y \in[0,1]}\left|W\left(1_{R(x, y)} f\right)\right|<\varepsilon\right\} \leq \mathcal{O}\left(\exp \left(-\frac{\left|f_{c}\right|(\log (1 / \varepsilon))^{3}}{C^{3} \varepsilon^{2}}\right)\right),
$$

where $C$ is a positive constant depending only on $m^{\prime}+n^{\prime}$.

For the proof let $\nu$ denote a positive measure on $[0,1]^{2}$. Let further be $W^{\nu}$ a centred Gaussian process with

$$
\mathbb{E}\left\{W^{\nu}(x, y) W^{\nu}\left(x^{\prime}, y^{\prime}\right)\right\}=\nu\left(\left[0, \min \left(x, x^{\prime}\right)\right] \times\left[0, \min \left(y, y^{\prime}\right)\right]\right) .
$$

Proof of Corollary 2.7. Set $A=\operatorname{supp}\left(h_{m^{\prime}, k^{\prime}} \otimes h_{n^{\prime}, l^{\prime}}\right)$, then

$$
f^{2}=\left(\left(f^{2}-f_{c}\right) \cdot 1_{A}+f^{2} \cdot 1_{A^{c}}\right)+f_{c} \cdot 1_{A}
$$

Moreover, set $u=\left(\left(f^{2}-f_{c}\right) \cdot 1_{A}+f^{2} \cdot 1_{A^{c}}\right)^{1 / 2}$ and $v=\left(f_{c} \cdot 1_{A}\right)^{1 / 2}$; the function $v$ satisfies the assumption of Proposition 2.6. Define for an arbitrary function $w \in L^{2}[0,1]^{2}$ the measure $\nu_{w}$ on $\mathcal{B}[0,1]^{2}$ by $\nu_{w}(B)=\int_{B} w(x)^{2} d x$. Then the two measures $\nu_{u}$ and $\nu_{v}$ are positive and $\nu_{u}+\nu_{v}=\nu_{f}$ by definition. 
Consider the three centred Gaussian processes $W^{\nu_{f}}, W^{\nu_{u}}$ and $W^{\nu_{v}}$ and assume that the last two are independent. One has

$$
\operatorname{law}\left(W^{\nu_{f}}\right)=\operatorname{law}\left(W^{\nu_{u}+\nu_{v}}\right)=\operatorname{law}\left(W^{\nu_{u}}+W^{\nu_{v}}\right) .
$$

and $W^{\nu_{u}}$ and $W^{\nu_{v}}$ can be considered as centred Gaussian random variables with values in $\left(C[0,1]^{2},\|\cdot\|_{\infty}\right)$. They are assumed to be independent and thus Lemma 2.1, [12] yields

$$
\mathbb{P}\left\{\left\|W^{\nu_{u}}+W^{\nu_{v}}\right\|_{\infty}<\varepsilon\right\} \leq \mathbb{P}\left\{\left\|W^{\nu_{v}}\right\|_{\infty}<\varepsilon\right\}
$$

for $\varepsilon>0$. Furthermore, the processes $W^{\nu_{f}}$ and $\left\{W\left(1_{R(x, y)} f\right) ;(x, y) \in\right.$ $\left.[0,1]^{2}\right\}$ are equal in law. Similarly, the processes $W^{\nu_{v}}$ and $\left\{W\left(1_{R(x, y)} v\right) ;(x, y) \in\right.$ $\left.[0,1]^{2}\right\}$ have the same law. Thus we can conclude

$$
\mathbb{P}\left\{\sup _{x, y \in[0,1]}\left|W\left(1_{R(x, y)} f\right)\right|<\varepsilon\right\} \leq \mathbb{P}\left\{\sup _{x, y \in[0,1]}\left|W\left(1_{R(x, y)} v\right)\right|<\varepsilon\right\}
$$

for $\varepsilon>0$. The assertion follows by application of Proposition 2.6.

Corollary 2.7 holds for each $f \in L^{2}[0,1]^{2}$ such that $f^{2}$ is bounded away from zero at least on a rectangle. For example, this is satisfied, if $f$ is continuous and does not vanish at at least one point. Hence, the important case of continuous functions which do not vanish identically is covered.

Definition 2.8 Let $B \subset \mathbb{R}^{2}$. Let $\mathcal{C} \mathcal{R}(B)$ denote the set

$\left\{f \in L^{2}(B) ; \exists Q=[a, b] \times[c, d] \subset B, f_{c}>0\right.$ with $\left.\left(f^{2}-f_{c}\right)\right|_{Q}>0$ a.e. $\}$.

Corollary 2.7 can still be improved.

Remark 2.9 Let $\nu$ be a positive and non singular measure on $[0,1]^{2}$ and let $W^{\nu}$ be a centred Gaussian process as in (10) before. Then Theorem 4.2, [12] yields for $\varepsilon \rightarrow 0+$

$$
\mathbb{P}\left\{\sup _{x, y \in[0,1]}\left|W^{\nu}(x, y)\right|<\varepsilon C(\nu)\right\} \leq \mathcal{O}\left(\exp \left(-\frac{(\log (1 / \varepsilon))^{3}}{C(\nu) \varepsilon^{2}}\right)\right),
$$

where $C(\nu)$ is a positive constant depending only on $\nu$. Let $f \in L^{2}[0,1]^{2}$ and define $\nu(A)=\int_{A} f(z)^{2} d z$ for $A \in \mathcal{B}[0,1]^{2}$. It is straightforward that $\nu$ is a positive and non singular measure on $[0,1]^{2}$. Moreover, $\left\{W\left(1_{R(x, y)} f\right) ;(x, y) \in\right.$ $\left.[0,1]^{2}\right\}$ is a centred Gaussian process with

$$
\mathbb{E}\left\{W\left(1_{R(x, y)} f\right) W\left(1_{R\left(x^{\prime}, y^{\prime}\right)} f\right)\right\}=\nu\left(\left[0, \min \left(x, x^{\prime}\right)\right] \times\left[0, \min \left(y, y^{\prime}\right)\right]\right) .
$$

Thus TALAGRAND's theorem yields

$$
\mathbb{P}\left\{\sup _{x, y \in[0,1]}\left|W\left(1_{R(x, y)} f\right)\right|<\varepsilon\right\} \leq \mathcal{O}\left(\exp \left(-\frac{C(f)(\log (1 / \varepsilon))^{3}}{\varepsilon^{2}}\right)\right),
$$

as $\varepsilon \rightarrow 0+$, where $C(f)$ depends only on $f$. Notice that the only restriction on $f$ is its square integrability. 
Our aim is to derive small ball asymptotics for solutions of a certain class of stochastic wave equations. The simplest stochastic wave equation on a real line is

$$
\frac{\partial^{2} X}{\partial t^{2}}(t, x)-\frac{\partial^{2} X}{\partial x^{2}}(t, x)=d W(t, x)
$$

with zero initial conditions. We define

$$
\triangle(t, x)=\left\{(s, y) \in \mathbb{R}_{+} \times \mathbb{R}: 0 \leq s \leq t,|x-y| \leq t-s\right\}
$$

and let

$$
G(t, x, s, y)=1_{\triangle(t, x)}(s, y) / 2
$$

be GREen's function for the wave equation on the real line. The solution of (11) can be written as $\left\{W(G(t, x)) ;(t, x) \in \mathbb{R}_{+} \times \mathbb{R}\right\}$; see for example [13] or [2]. By rotation and shift, we establish a link to the process $\left\{W\left(1_{R(x, y)}\right) ;(t, x) \in \mathbb{R}_{+} \times \mathbb{R}\right\}$ for which we derived asymptotics before.

A point $z \in[0,1]^{2}$ is transferred to a point $(t, x) \in \mathbb{R}_{+} \times \mathbb{R}$ by a $45^{\circ}$ clockwise rotation and a shift by $\left(-2^{-1 / 2}, 0\right)$, i.e.

$$
(t, x)=\left(\frac{z_{1}+z_{2}}{\sqrt{2}}, \frac{-z_{1}+z_{2}}{\sqrt{2}}\right)-\left(\frac{1}{\sqrt{2}}, 0\right)
$$

equivalently

$$
z=\left(\frac{t+2^{-1 / 2}-x}{\sqrt{2}}, \frac{t+2^{-1 / 2}+x}{\sqrt{2}}\right) .
$$

Let the clockwise rotation with shift be denoted by $r^{-}$and the shift with anti-clockwise rotation be denoted by $r^{+}$.

Corollary 2.10 Let $f \in \mathcal{C} \mathcal{R}\left(\triangle\left(2^{-1 / 2}, 0\right)\right)$, then

$$
\mathbb{P}\left\{\sup _{(x, t) \in \triangle\left(2^{-1 / 2}, 0\right)}|W(G(t, x) f)|<\varepsilon\right\} \leq \mathcal{O}\left(\exp \left(-\frac{C(f)(\log (1 / \varepsilon))^{3}}{4 \varepsilon^{2}}\right)\right)
$$

for $\varepsilon \rightarrow 0+$, where $C(f)$ is a constant depending only on $f$.

Proof. Define the function $f_{r^{-}}$on $[0,1]^{2}$ by

$$
f_{r^{-}}(z)= \begin{cases}f\left(r^{-}(z)\right) & \text { if } z_{1}+z_{2} \geq 1 \\ 0 & \text { otherwise }\end{cases}
$$

Then the two random variables $W(G(t, x) f)$ and $W\left((1 / 2) 1_{R_{z}} f_{r^{-}}\right)$are equal in law for $z=r^{+}(t, x)$. Thus

$$
\sup _{\triangle\left(2^{-1 / 2}, 0\right)}|W(G(t, x) f)| \quad \text { and } \quad \sup _{[0,1]^{2}}\left|W\left((1 / 2) 1_{R_{z}} f_{r^{-}}\right)\right|
$$


are equal in law and the assertion follows from Corollary 2.7.

Putting things together gives the main result of this section. Notice that we use for the partial differential equations in question their integral equation representation, too; see again [13] or [2].

Theorem 2.11 Let $f \in L^{2}\left(\mathbb{R}_{+} \times \mathbb{R}\right)$ such that $\left.f\right|_{\triangle\left(2^{-1 / 2}, 0\right)} \in \mathcal{C} \mathcal{R}\left(\triangle\left(2^{-1 / 2}, 0\right)\right)$ and let $g$ be a LIPSCHITZ continuous function on $\mathbb{R}$. Let $u_{0}$ be a continuous function from $\mathbb{R}_{+} \times \mathbb{R}$ to $\mathbb{R}$ and let $X$ be the solution of the stochastic integral equation

$$
X(t, x)=u_{0}(t, x)+\int_{\mathbb{R}_{+}} \int_{\mathbb{R}} G(t, x, s, y) g(X(s, y)) d y d s+W(G(t, x) f)
$$

simultaneously for $(t, x) \in \mathbb{R}_{+} \times \mathbb{R}$, almost surely. Finally, let $u$ be the solution of the deterministic integral equation

$$
u(t, x)=u_{0}(t, x)+\int_{\mathbb{R}_{+}} \int_{\mathbb{R}} G(t, x, s, y) g(u(s, y)) d y d s .
$$

Then for $\varepsilon \rightarrow 0+$,

$$
\mathbb{P}\left\{\sup _{\triangle\left(2^{-1 / 2}, 0\right)}|X(t, x)-u(t, x)|<\varepsilon\right\} \leq \mathcal{O}\left(\exp \left(-\frac{C(f)(\log (1 / \varepsilon))^{3}}{4\left(1+C_{L}(g) / 4\right)^{2} \varepsilon^{2}}\right)\right) .
$$

Proof. Rewriting the subtraction of (14) from (13) and taking absolute values yields

$$
\begin{aligned}
& |W(G(t, x) f)| \\
& \quad \leq|X(t, x)-u(t, x)|+\int_{\mathbb{R}_{+}} \int_{\mathbb{R}} G(t, x, s, y)|g(X(s, y))-g(u(s, y))| d y d s .
\end{aligned}
$$

Since $g$ is LIPSCHITZ continuous with LIPSCHITZ constant $C_{L}(g)$, the last expression is less or equal to

$$
\begin{aligned}
|X(t, x)-u(t, x)| & +C_{L}(g) \int_{\mathbb{R}_{+}} \int_{\mathbb{R}} G(t, x, s, y)|X(s, y)-u(s, y)| d y d s \\
& \leq \sup _{(x, t) \in \triangle\left(2^{-1 / 2}, 0\right)}|X(t, x)-u(t, x)|\left(1+\frac{C_{L}(g) 1 / 2}{2}\right)
\end{aligned}
$$

simultaneously for all $(t, x) \in \triangle\left(2^{-1 / 2}, 0\right)$ with probability one. Furthermore, if $\sup _{\triangle\left(2^{-1 / 2}, 0\right)}|X(t, x)-u(t, x)|<\varepsilon$, then this implies

$$
\sup _{\triangle\left(2^{-1 / 2}, 0\right)}|W(G(t, x) f)|<\varepsilon\left(1+C_{L}(g) / 4\right),
$$


and thus for $\varepsilon \rightarrow 0+$,

$$
\begin{aligned}
& \mathbb{P}\left\{\sup _{\triangle\left(2^{-1 / 2}, 0\right)}|X(t, x)-u(t, x)|<\varepsilon\right\} \\
& \quad \leq \mathbb{P}\left\{\sup _{(x, t) \in \triangle\left(2^{-1 / 2}, 0\right)}|W(G(t, x) f)|<\varepsilon\left(1+C_{L}(g) / 4\right)\right\} \\
& \quad \leq \mathcal{O}\left(\exp \left(-\frac{C(f)(\log (1 / \varepsilon))^{3}}{4\left(1+C_{L}(g) / 4\right)^{2} \varepsilon^{2}}\right)\right) .
\end{aligned}
$$

As announced, we discuss the KLEIN-GORDON equation perturbed by spacetime white noise.

Example 2.12 Consider the stochastic KLEIN-Gordon equation

$$
\frac{\partial^{2} X}{\partial t^{2}}(t, x)-\frac{\partial^{2} X}{\partial x^{2}}(t, x)=\alpha X(t, x)+d W(t, x)
$$

with initial conditions $(F, \mu)$. Let $u$ be the solution of the deterministic KLEIN-GORDON equation

$$
\frac{\partial^{2} X}{\partial t^{2}}(t, x)-\frac{\partial^{2} X}{\partial x^{2}}(t, x)=\alpha X(t, x)
$$

with initial conditions $(F, \mu)$. Then Proposition 2.11 yields that for $\varepsilon \rightarrow 0+$

$$
\mathbb{P}\left\{\sup _{\triangle\left(2^{-1 / 2}, 0\right)}|X(t, x)-u(t, x)|<\varepsilon\right\} \leq \mathcal{O}\left(\exp \left(-\frac{(\log (1 / \varepsilon))^{3}}{4(1+\alpha / 4)^{2} C_{0}^{3} \varepsilon^{2}}\right)\right) .
$$

On the other hand the solution of equation (15) is given by

$$
X(t, x)=j_{F, \mu}(t, x)+\int_{\mathbb{R}_{+}} \int_{\mathbb{R}} G_{K G}^{\alpha}(t, x, s, y) d W(s, y),
$$

where

$$
G_{K G}^{\alpha}(t, x, s, y)=G(t, x, s, y) I_{0}\left(\sqrt{\alpha\left((t-s)^{2}-(x-y)^{2}\right)}\right) ; \alpha \geq 0
$$

and

$$
G_{K G}^{\alpha}(t, x, s, y)=G(t, x, s, y) J_{0}\left(\sqrt{|\alpha|\left((t-s)^{2}-(x-y)^{2}\right)}\right) ; \alpha<0,
$$

respectively. $I_{0}$ is the modified Bessel function of order zero and of the first kind and $J_{0}$ is the regular Bessel function of order zero and of the first kind. The function $j_{F, \mu}$ is defined by

$$
j_{F, \mu}(t, x)=\frac{\partial}{\partial t} \int_{x-t}^{x+t} G_{K G}^{\alpha}(t, x, 0, y) F(y) d y+\int_{x-t}^{x+t} G_{K G}^{\alpha}(t, x, 0, y) \mu(d y) .
$$


Moreover, $j_{F, \mu}$ is the solution of the deterministic homogeneous KLEINGordon equation (16) which yields $j_{F, \mu}=u$. Thus inequality (17) yields a small ball asymptotics for (18), i.e. for $\varepsilon \rightarrow 0+$

$\mathbb{P}\left\{\sup _{\triangle\left(2^{-1 / 2}, 0\right)}\left|X(t, x)-j_{F, \mu}(t, x)\right|<\varepsilon\right\} \leq \mathcal{O}\left(\exp \left(-\frac{(\log (1 / \varepsilon))^{3}}{4(1+\alpha / 4)^{2} C_{0}^{3} \varepsilon^{2}}\right)\right)$.

In the case $\alpha=0$, equation (18) reduces to the solution of the linear stochastic wave equation.

\section{Lower Asymptotics}

In this section we derive the small ball asymptotics from below. We start from TALAGRAND's general result for a Brownian sheet and transfer this asymptotics to our special case of stochastic wave equations. We give the main result of this section in Theorem 3.4. Finally, we discuss again the example of the stochastic KLEIN-GORDON equation.

Let $\nu$ be a positive measure on $[0,1]^{2}$ and let $W^{\nu}$ denote again a centred Gaussian process on $[0,1]^{2}$ with

$$
\mathbb{E}\left\{W^{\nu}(x, y) W^{\nu}\left(x^{\prime}, y^{\prime}\right)\right\}=\nu\left(\left[0, \min \left(x, x^{\prime}\right)\right] \times\left[0, \min \left(y, y^{\prime}\right)\right]\right) .
$$

The analogue of Corollary 2.7 reads:

Proposition 3.1 ([12], Proposition 4.1) For $\varepsilon \rightarrow 0+$,

$$
\mathbb{P}\left\{\sup _{x, y \in[0,1]}\left|W^{\nu}(x, y)\right|<\left.\varepsilon\|\nu\|\right|^{1 / 2}\right\} \geq \mathcal{O}\left(\exp \left(-\frac{(\log (1 / \varepsilon))^{3}}{\varepsilon^{2}}\right)\right) .
$$

Let $f \in L^{2}[0,1]^{2}$ with $\|f\|_{2}>0$. Consider the positive and non singular measure $\nu$ on $\mathcal{B}[0,1]^{2}$ defined by $\nu(A)=\int_{A} f(z)^{2} d z$. Then $\left\{W\left(1_{R(x, y)} f\right) ;(x, y) \in\right.$ $\left.[0,1]^{2}\right\}$ is a centred Gaussian process with

$$
\mathbb{E}\left\{W\left(1_{R(x, y)} f\right) W\left(1_{R\left(x^{\prime}, y^{\prime}\right)} f\right)\right\}=\nu\left(\left[0, \min \left(x, x^{\prime}\right)\right] \times\left[0, \min \left(y, y^{\prime}\right)\right]\right)
$$

and Proposition 3.1 yields for $\varepsilon \rightarrow 0+$,

$$
\mathbb{P}\left\{\sup _{x, y \in[0,1]}\left|W\left(1_{R(x, y)} f\right)\right|<\varepsilon\right\} \geq \mathcal{O}\left(\exp \left(-\frac{\|f\|_{2}(\log (1 / \varepsilon))^{3}}{\varepsilon^{2}}\right)\right) .
$$

The next step is rotating and shifting coordinates like in the section above. Recall that the $45^{\circ}$-clockwise rotation with shift is denoted by $r^{-}$and the shift with anti-clockwise rotation is denoted by $r^{+}$.

Corollary 3.2 Let $f \in L^{2}\left(\triangle\left(2^{-1 / 2}, 0\right)\right)$ with $\|f\|_{2}>0$. Then for $\varepsilon \rightarrow 0+$,

$$
\mathbb{P}\left\{\sup _{\triangle\left(2^{-1 / 2}, 0\right)}|W(G(t, x) f)|<\varepsilon\right\} \geq \mathcal{O}\left(\exp \left(-\frac{\|f\|_{2}(\log (1 / \varepsilon))^{3}}{4 \varepsilon^{2}}\right)\right) .
$$


Proof. Define the function $f_{r^{-}}$on $[0,1]^{2}$ by

$$
f_{r^{-}}(z)= \begin{cases}f\left(r^{-}(z)\right) & \text { if } z_{1}+z_{2} \geq 1 \\ 0 & \text { otherwise. }\end{cases}
$$

Then the two random variables $W(G(t, x) f)$ and $W\left((1 / 2) 1_{R_{z}} f_{r^{-}}\right)$are equal in law for $z=r^{+}(t, x)$ and hence

$$
\sup _{\triangle\left(2^{-1 / 2}, 0\right)}|W(G(t, x) f)| \text { and } \sup _{[0,1]^{2}}\left|W\left((1 / 2) 1_{R_{z}} f_{r^{-}}\right)\right|
$$

are equal in law, too. This implies the assertion.

Recall now Gronwall's lemma [14], Proposition 19. III, p. 125: Let the order interval $\{y \preceq z\}$ be given by

$$
\{y \preceq z\}=\left\{y \in \mathbb{R}_{+}^{d} ; 0 \leq y_{i} \leq z_{i}, \text { for all } i \in\{1, \ldots, d\}\right\}
$$

and denote the closure of a set $G \in \mathbb{R}_{+}^{d}$ by $\bar{G}$.

Lemma 3.3 (Gronwall) Let $G \in \mathbb{R}_{+}^{d}, \alpha \in \mathbb{R}, \beta>0$ and let $v \in C(\bar{G})$ with

$$
v(z) \leq \alpha+\beta \int_{\bar{G} \cap\{y \preceq z\}} v(y) d y .
$$

Then

$$
v(z) \leq \alpha E_{d}\left(\beta z_{1} \cdots z_{d}\right):=\alpha \sum_{i=0}^{\infty} \frac{\left(\beta z_{1} \cdots z_{d}\right)^{i}}{(i !)^{d}} .
$$

Especially, $E_{1}(x)=\exp (x)$ and $E_{2}(x)=I_{0}(2 \sqrt{x})$, where $I_{0}$ denotes the modified Bessel function of order zero and of the first kind.

Theorem 3.4 Let $f \in L^{2}\left(\mathbb{R}_{+} \times \mathbb{R}\right)$ with $\left\|f 1_{\triangle\left(2^{-1 / 2}, 0\right)}\right\|_{2}>0$ and let $g$ be a LIPSCHITZ continuous function on $\mathbb{R}$. Further, let $u_{0}$ be a continuous function from $\mathbb{R}_{+} \times \mathbb{R}$ to $\mathbb{R}$ and let $X$ be the solution of the stochastic integral equation

$$
X(t, x)=u_{0}(t, x)+\int_{\mathbb{R}_{+}} \int_{\mathbb{R}} G(t, x, s, y) g(X(s, y)) d y d s+W(G(t, x) f)
$$

simultaneously for $(t, x) \in \mathbb{R}_{+} \times \mathbb{R}$, almost surely. Finally, let $u$ be the solution of the deterministic integral equation

$$
u(t, x)=u_{0}(t, x)+\int_{\mathbb{R}_{+}} \int_{\mathbb{R}} G(t, x, s, y) g(u(s, y)) d y d s .
$$

Then for $\varepsilon \rightarrow 0+$,

$$
\begin{aligned}
& \mathbb{P}\left\{\sup _{\triangle\left(2^{-1 / 2}, 0\right)}|X(t, x)-u(t, x)|<\varepsilon\right\} \\
& \quad \geq \mathcal{O}\left(\exp \left(-\frac{\|f\|_{2} I_{0}\left(\sqrt{2 C_{L}(g)}\right)(\log (1 / \varepsilon))^{3}}{4 \varepsilon^{2}}\right)\right) .
\end{aligned}
$$


Proof. The following inequalities hold almost surely. Suppose that

$$
\sup _{\triangle\left(2^{-1 / 2}, 0\right)}|W(G(t, x) f)| \leq \varepsilon / I_{0}\left(2 \sqrt{C_{L}(g)} / \sqrt{2}\right) .
$$

With the integral equations (19) and (20), and the triangle inequality one achieves for each $(t, x) \in \triangle\left(2^{-1 / 2}, 0\right)$,

$$
\begin{aligned}
& |X(t, x)-u(t, x)| \\
& \quad \leq \int_{\mathbb{R}_{+}} \int_{\mathbb{R}} G(t, x, s, y)|g(X(s, y))-g(u(s, y))| d y d s+|W(G(t, x) f)| \\
& \quad \leq \frac{C_{L}(g)}{2} \iint_{\triangle\left(2^{-1 / 2}, 0\right)}|X(s, y)-u(s, y)| d y d s+\varepsilon / I_{0}\left(2 \sqrt{C_{L}(g)} / \sqrt{2}\right) .
\end{aligned}
$$

In the last inequality the LIPSCHITZ property of $g$ is used. In the notation with shifted and rotated coordinates this is equal to

$$
|X(z)-u(z)| \leq \frac{C_{L}(g)}{2} \int_{R(z)}|X(w)-u(w)| d w+\varepsilon / I_{0}\left(2 \sqrt{C_{L}(g)} / \sqrt{2}\right),
$$

where $X(z)=u(z)=0$ for $z_{1}+z_{2}<1$. GronwaLL's lemma yields

$$
|X(z)-u(z)| \leq \frac{\varepsilon}{I_{0}\left(2 \sqrt{C_{L}(g)} / \sqrt{2}\right)} I_{0}\left(2 \sqrt{\left(C_{L}(g) / 2\right) z_{1} z_{2}}\right) \leq \varepsilon,
$$

for $z \in[0,1]^{2}$ and thus

$$
\sup _{\triangle\left(2^{-1 / 2}, 0\right)}|X(t, x)-u(t, x)|=\sup _{[0,1]^{2}}|X(z)-u(z)| \leq \varepsilon .
$$

This implies for $\varepsilon \rightarrow 0+$ that

$$
\begin{aligned}
& \mathbb{P}\left\{\sup _{(x, t) \in \triangle\left(2^{-1 / 2}, 0\right)}|X(t, x)-u(t, x)|<\varepsilon\right\} \\
& \geq \mathbb{P}\left\{\sup _{(x, t) \in \triangle\left(2^{-1 / 2}, 0\right)}|W(G(t, x) f)|<\varepsilon / I_{0}\left(2 \sqrt{C_{L}(g)} / \sqrt{2}\right)\right\} \\
& \geq \mathcal{O}\left(\exp \left(-\frac{\|f\|_{2} I_{0}\left(\sqrt{2 C_{L}(g)}\right)(\log (1 / \varepsilon))^{3}}{4 \varepsilon^{2}}\right)\right)
\end{aligned}
$$

and the proof is complete.

The example is again the KLEIN-GORDON equation perturbed by space-time white noise.

Example 3.5 Consider the stochastic KLEIN-Gordon equation

$$
\frac{\partial^{2} X}{\partial t^{2}}(t, x)-\frac{\partial^{2} X}{\partial x^{2}}(t, x)-\alpha X(t, x)=d W(t, x)
$$


with initial conditions $(F, \mu)$. The lower asymptotics can be derived like in Example 2.12, and for $\varepsilon \rightarrow 0+$

$$
\begin{aligned}
& \mathbb{P}\left\{\sup _{\triangle\left(2^{-1 / 2}, 0\right)}\left|X(t, x)-j_{F, \mu}(t, x)\right|<\varepsilon\right\} \\
& \quad \geq \mathcal{O}\left(\exp \left(-\frac{I_{0}(\sqrt{2|\alpha|})(\log (1 / \varepsilon))^{3}}{4 \varepsilon^{2}}\right)\right),
\end{aligned}
$$

where again

$$
j_{F, \mu}(t, x)=\frac{\partial}{\partial t} \int_{x-t}^{x+t} G_{K G}^{\alpha}(t, x, 0, y) F(y) d y+\int_{x-t}^{x+t} G_{K G}^{\alpha}(t, x, 0, y) \mu(d y) .
$$

Acknowledgement. I like to thank Volkmar Liebscher, Peter Mörters, Gerhard Winkler, and Olaf Wittich for stimulating discussions and suggestions. I am also indebted to Robert C. Dalang who draw my attention to this subject.

\section{References}

[1] R. Carmona and D. Nualart. Random nonlinear wave equations: Propagation of singularities. Ann. Probab., 16(2):730-751, 1988.

[2] R. Carmona and D. Nualart. Random nonlinear wave equations: Smoothness of the solutions. Probab. Theory Relat. Fields, 79(4):469$508,1988$.

[3] J.P.R. Christensen. A survey of small ball theorems and problems. In Measure theory, Proc. Conf., Oberwolfach 1979, Lect. Notes Math. 794, 24-30 . 1980.

[4] G. DaPrato and J. Zabczyk. Stochastic equations in infinite dimensions. Encyclopedia of Mathematics and Its Applications. 44. Cambridge etc.: Cambridge University Press. xviii, 454 p. , 1992.

[5] H. Holden, B. Øksendal, J. Ubøe, and T. Zhang. Stochastic partial differential equations. A modeling, white noise functional approach. Probability and Its Applications. Basel: Birkhäuser. 230 p. , 1996.

[6] J. Kuelbs and W.V. Li. Metric entropy and the small ball problem for Gaussian measures. J. Funct. Anal., 116(1):133-157, 1993.

[7] M. Ledoux. Isoperimetry and Gaussian analysis. In Dobrushin, $R$. (ed.) et al., Lectures on probability theory and statistics. Ecole d'été de probabilités de Saint-Flour XXIV-1994. Lectures given at the summer school in Saint-Flour, France, July 7-23, 1994. Berlin: Springer. Lect. Notes Math. 1648, 165-294. 1996. 
[8] M. Ledoux and M. Talagrand. Probability in Banach spaces. Isoperimetry and processes. Ergebnisse der Mathematik und ihrer Grenzgebiete. 3. Folge, 23. Berlin etc.: Springer-Verlag. xii, 480 p. , 1991.

[9] W.V. Li and W. Linde. Approximation, metric entropy and small ball estimates for Gaussian measures. Ann. Probab., 27(3):1556-1578, 1999.

[10] W.V. Li and Q.M. Shao. Gaussian processes: Inequalities, small ball probabilities and applications. In Shanbhag, D. N. (ed.) et al., Stochastic processes: Theory and methods. Amsterdam: North-Holland/ Elsevier. Handb. Stat. 19, 533-597. 2001.

[11] M.A. Lifshits. Asymptotic behavior of small ball probabilities. In Grigelionis, B. (ed.) et al., Probability theory and mathematical statistics. Proceedings of the 7th international Vilnius conference, Vilnius, Lithuania, August, 12-18, 1998. Vilnius: TEV. 453-468. 1999.

[12] M. Talagrand. The small ball problem for the Brownian sheet. Ann. Probab., 22(3):1331-1354, 1994.

[13] J.B. Walsh. An introduction to stochastic partial differential equations. In École d'été de probabilités de Saint-Flour XIV - 1984, Lect. Notes Math. 1180, 265-43\%. 1986.

[14] W. Walter. Differential- und Integral-Ungleichungen und ihre Anwendung bei Abschätzungs- und Eindeutigkeitsproblemen. Ergebnisse der angewandten Mathematik. 2. Berlin-Göttingen-Heidelberg-New York: Springer-Verlag. XIII, 269 S. mit 18 Abb., 1964. 